\title{
New Baxter phase in the Ashkin-Teller model on a cubic lattice
}

\author{
J.P. Santos ${ }^{a, b, *}$, D.S. Rosa ${ }^{c}$, F.C. Sá Barreto ${ }^{a, d}$ \\ a Departamento de Ciências Naturais, Universidade Federal de São João del Rei, C.P. 110, 36301-160, São João del Rei, MG, Brazil \\ b Departamento de Matemática, Universidade Federal de São João del Rei, C.P. 110, 36301-160, São João del Rei, MG, Brazil \\ c Instituto de Física Teórica, Universidade Estadual Paulista, C.P. 110, 01140-070, São Paulo, SP, Brazil \\ d Departamento de Física, Universidade Federal de Minas Gerais, C.P. 110, 31270-901, Belo Horizonte, MG, Brazil ${ }^{1}$
}

\section{A R T I C L E I N F O}

\section{Article history:}

Received 11 September 2017

Received in revised form 9 November 2017

Accepted 23 November 2017

Available online 28 November 2017

Communicated by F. Porcelli

\section{Keywords:}

Ashkin-Teller model

New Baxter phase

Cubic lattice

\begin{abstract}
A B S T R A C T
The mean field theory results are obtained from the Bogoliubov inequality for the spin- $1 / 2$ Ashkin-Teller model on a cubic lattice for different cluster sizes. The phase diagram, magnetization and free energy are obtained. From those expressions we observed a new phase in the model. Denoted in the course of this work by Baxter ${ }^{(2)}$ this new phase presents $\langle S\rangle \neq\langle\sigma\rangle \neq 0$. The phase transitions between the Baxter ${ }^{(2)}$ and the others well known phases for the model are studied and classified.
\end{abstract}

(C) 2017 Elsevier B.V. All rights reserved.

\section{Introduction}

Despite being massively studied for decades, the Ashkin-Teller (AT) model [1] still attracts a great interest due its application in different areas such as magnetism [2], chemical interactions in metallic alloys [3], elastic response of DNA molecule to external force and torque [4] and phase diagram of selenium adsorbed on the $\mathrm{Ni}(100)$ surface [5]. The AT model due to the presence of the tricritical points and weakly first order phase transitions presents a complicated and interesting phase diagram. The properties of the AT model were studied by a variety of methods such as mean-field theory (MFT) [6-8], effective field theory (EFT) [9], rigorous inequality correlation function [10], renormalization group theory (RG) [11,12], mean-field renormalization group (MFRG) $[13,14]$ and Monte Carlo methods (MC) [6, 15-21].

In the context of short series analysis, MC simulation, MFT and RG Ditzian et al. [6] studied the AT model in three dimensions. There, the authors found what seems to be a new phase where $\langle S\rangle \neq\langle\sigma\rangle \neq 0$. This unexpected behavior was observed in the MC context for different lattice sizes; in this same work the new phase

\footnotetext{
* Corresponding author at: Departamento de Ciências Naturais, Universidade Federal de São João del Rei, C.P. 110, 36301-160, São João del Rei, MG, Brazil.

E-mail addresses: jander@ufsj.edu.br (J.P. Santos), derick@ift.unesp.br (D.S. Rosa), fcsabarreto@gmail.com (F.C. Sá Barreto).

1 Emeritus Professor.
}

was not observed in MFT context. In Ref. [6] it is also possible to find a discussion of the AT model results obtained from MFT in two and three dimensions. Later the results by Ditzian obtained from the MC simulations for the AT model in 3D were confirmed by Musial [18].

In this work we obtained the MFT results from the Bogoliubov inequality for the spin-1/2 AT model on a cubic lattice for clusters with 1-, 2-, 4-, and 8-sites (see Fig. 1) in order to find and study for the first time the new phase present in the model. In this work the new phase will be labeled by Baxter ${ }^{(2)}$. Phase transitions between Baxter ${ }^{(2)}$ and the others well know phases already mentioned in previous works for the AT model are obtained and classified. The MFT approximation is used in this work as a especially tool suitable for a first view into the problem. Although we have historically observed that the MFT approach does not provide accurate results compared to other numerical and analytical methods, we can say that this is a good approach for a first qualitative insight into the problem.

The paper is organized as follows. The section 2 is devoted to obtain the MFT results from the Bogoliubov inequality for the spin-1/2 AT model. Expressions for the free energy and magnetization were computed. For clarity analytical results for the cluster with 1-site are presented. In section 3, we present the results of the phase diagrams, magnetizations $\left(\langle S\rangle=m_{S},\langle\sigma\rangle=m_{\sigma}\right.$ and $\left.\langle S \sigma\rangle=m_{\sigma S}\right)$ and free energy in order to find the $\operatorname{Baxter}^{(2)}$ phase and classify its transitions with the other phases already known for the AT model. Our final comments are presented in section 4. 


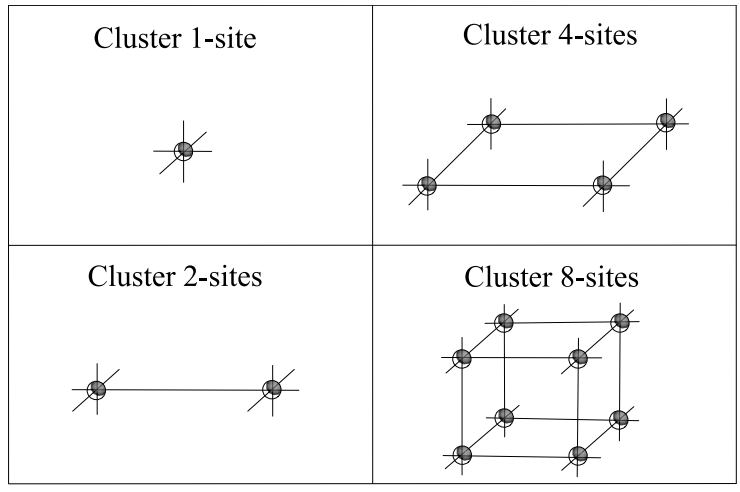

Fig. 1. Schematic representations of a cubic lattice structure for cluster with 1-, 2-, 4- and 8-sites.

\section{Model and formulation}

In this section we show the formalism used to solve the spin-1/2 AT model applying the MFT from the Bogoliubov inequality [22-24]. We obtain phase diagrams, magnetization and free energy. Equations for 1-site cluster in a cubic lattice are presented.

The AT model [1] is a generalization of the Ising model to a four-component system. It may be considered as two superposed Ising models described by variables $S_{i}$ and $\sigma_{i}$ sitting on sites of a lattice. For each Ising model there is a two-spin nearest-neighbor interaction $J$ and the two different Ising models are coupled by a four-spin interaction $K$. The Hamiltonian can then be written as

$H=-\sum_{<i j>}\left[J\left(S_{i} S_{j}+\sigma_{i} \sigma_{j}\right)+K S_{i} \sigma_{i} S_{j} \sigma_{j}\right]$,

where $<i j>$ denotes a pair of nearest-neighbor spins.

The Bogoliubov variational principle [22-24] is based on a validity of the inequality

$G \leq \phi=G_{0}+\left\langle H-H_{0}\right\rangle_{0}$,

where $G$ and $H$ represent the true Gibbs free energy and the full Hamiltonian for a considered lattice-statistical model and $G_{0}$ and $H_{0}$ represent the trial Gibbs free energy and the trial Hamiltonian of a simplified lattice-statistical model for which the relevant calculations can be performed exactly (the symbol $\langle\ldots\rangle_{0}$ denotes canonical ensemble average within the simplified model defined by the Hamiltonian $H_{0}$ ). The expression $\phi$ represents the variational Gibbs free energy, which provides an upper bound for the true Gibbs free energy.

In the context of MFT the Hamiltonian of a single spin is given by

$H_{i}=-\sum_{j}\left[J\left(S_{i}\left\langle S_{j}\right\rangle-\sigma_{i}\left\langle\sigma_{j}\right\rangle\right)-K \sigma_{i} S_{i}\left\langle\sigma_{j} S_{j}\right\rangle\right]$,

where $H_{i}$ is the Hamiltonian describing site $i$ and its neighbors $j$.

According to this we can write the trial Hamiltonian in the MFT for the spin-1/2 AT model with null external field as

$H_{0}=-\sum_{k}^{N}\left(S_{k} \gamma_{S}+\sigma_{k} \gamma_{\sigma}+\sigma_{k} S_{k} \gamma_{\sigma S}\right)$,

where $N$ denotes the total number of sites on the lattice. $H_{0}$ given by Eq. (4) depends on three different variational parameters $\gamma_{\tau}$ ( $\tau=\{S, \sigma, \sigma S\}$ ) and can be thought as an effective field acting on a spin in the frontier of a site $k$. The variable $\gamma$ has the physical meaning of an effective field.
The partition function $Z_{0}$, the Gibbs free energy $G_{0}$, the magnetization $m_{0}=m_{\tau}=\left\langle\tau_{i}\right\rangle_{0}$, are given by,

$$
\begin{aligned}
Z_{0} & =\sum_{\left\{\tau_{k}\right\}} \exp \left(-\beta H_{0}\right) \\
& =\prod_{k=1}^{N} \sum_{\tau_{k}= \pm 1} \exp \left[\beta\left(S_{k} \gamma_{S}+\sigma_{k} \gamma_{\sigma}+\sigma_{k} S_{k} \gamma_{\sigma s}\right)\right], \\
G_{0} & =-N k_{B} T \ln \left[4\left(\prod_{\{\tau\}} \cosh \beta \gamma_{\tau}+\prod_{\{\tau\}} \sinh \beta \gamma_{\tau}\right)\right], \\
m_{\tau} & =-\frac{1}{N} \frac{\partial G_{0}}{\partial \gamma_{\tau}} .
\end{aligned}
$$

From the mean value of the difference between the true and trial Hamiltonians $\left(\left\langle\mathrm{H}-\mathrm{H}_{0}\right\rangle_{0}\right)$ we obtain the expression for the variational Gibbs free energy. This quantity determines an upper bound for the true Gibbs free energy and is written as

$\phi=G_{0}-\frac{N z}{2}\left(J m_{S}^{2}+J m_{\sigma}^{2}+K m_{\sigma S}^{2}\right)+N \sum_{\{\tau\}} m_{\tau} \gamma_{\tau}$,

where the $z$ represents the coordination number of the lattice.

To study the stability of the model we obtain the critical points of the variational Gibbs free energy. The objective is to extremize the variational Gibbs free energy with respect to the variational parameters, i.e,

$$
\begin{aligned}
\frac{\partial \phi}{\partial \gamma_{\tau}}=0 \Leftrightarrow & \left(\gamma_{S}-z J m_{S}\right) \frac{\partial m_{S}}{\partial \gamma_{\tau}}+\left(\gamma_{\sigma}-z J m_{\sigma}\right) \frac{\partial m_{\sigma}}{\partial \gamma_{\tau}} \\
& +\left(\gamma_{\sigma S}-z K m_{\sigma S}\right) \frac{\partial m_{\sigma S}}{\partial \gamma_{\tau}}=0,
\end{aligned}
$$

which are simultaneously satisfied if the variational parameters are $\gamma_{S}=z J m_{S}, \gamma_{\sigma}=z J m_{\sigma}$ and $\gamma_{\sigma s}=z K m_{\sigma s}$.

All the formalism necessary to study the model was developed and now we are ready to find our numerical results. For clarity we present in the next two equations the analytical Gibbs free energy and magnetizations $\left(N m_{\tau}=-\partial G_{0} / \partial \gamma_{\tau}\right)$ obtained from the Bogoliubov inequality applied to the 1-site cluster of a cubic lattice $(z=6)$

$$
\begin{aligned}
G= & -N k_{B} T \ln \left[4\left(\prod_{\{\tau\}} \cosh \beta \gamma_{\tau}+\prod_{\{\tau\}} \sinh \beta \gamma_{\tau}\right)\right] \\
& +3 N\left[J m_{S}^{2}+J m_{\sigma}^{2}+K m_{\sigma S}^{2}\right], \\
m_{\tau}= & \frac{\tanh \beta \gamma_{\tau}+\tanh \beta \gamma_{\tau^{\prime}} \tanh \beta \gamma_{\tau^{\prime \prime}}}{1+\tanh \beta \gamma_{\tau} \tanh \beta \gamma_{\tau^{\prime}} \tanh \beta \gamma_{\tau^{\prime \prime}}} .
\end{aligned}
$$

The continuous phase transition line is obtained from the magnetization under the condition that $\left(m_{\tau} \rightarrow 0\right)$. The first-order transition line is determined from a comparison of the Gibbs free energies Eq. (10) from one ferromagnetic state with a spontaneous magnetization $m_{\tau}$ and another ferromagnetic state with a spontaneous magnetization $m_{\tau}^{\prime}$ both satisfying the 'self-consistent' Eq. (11), for the magnetization, i.e, $G\left(m_{\tau}\right)=G\left(m_{\tau}^{\prime}\right)$ with $m_{\tau} \neq m_{\tau}^{\prime}$.

The mean-field theory for the 2-, 4- and 8-sites clusters are similarly obtained using a generalization [25], where $H_{0}^{(n)}$ is as follows

$$
\begin{aligned}
H_{0}^{(n)}= & -\sum_{<\ldots>}^{N^{\prime}}\left[\sum_{<i j>}^{n}\left(J S_{i} S_{j}+J \sigma_{i} \sigma_{j}+K \sigma_{i} S_{i} \sigma_{j} S_{j}\right)\right. \\
& \left.+\sum_{i=1}^{n}\left(S_{i} \gamma_{1 i}+\sigma_{i} \gamma_{2 i}+\sigma_{i} S_{i} \gamma_{3 i}\right)\right],
\end{aligned}
$$




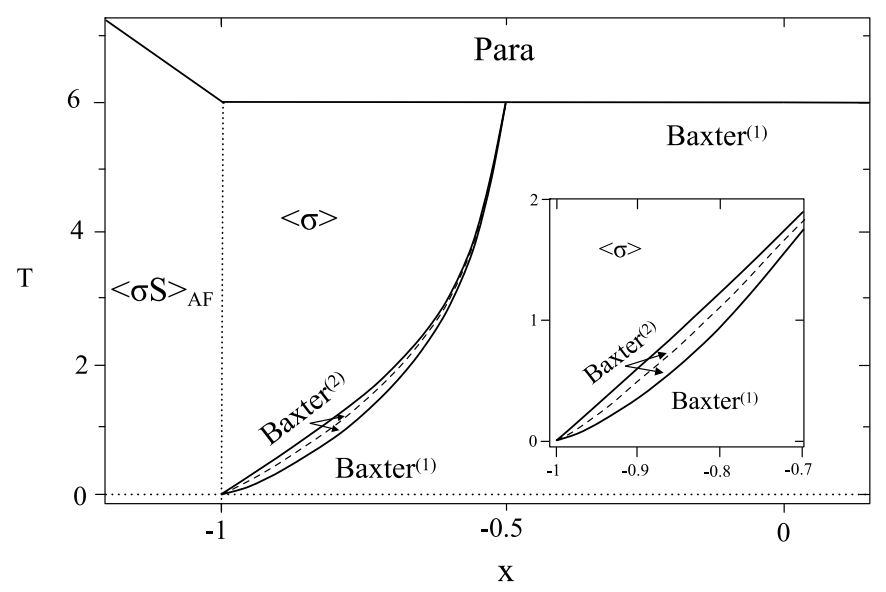

Fig. 2. The Phase diagram as a function of $x$ and $T$ for the spin- $1 / 2$ AT model in a cubic lattice with 1-site cluster obtained from MFT. Second order phase transitions are represented by solid lines. The first order transitions is represented by the dashed line. The dotted lines represent the value of the zero temperature and the boundary between the phases $\langle\sigma S\rangle_{A F}$ and $\langle\sigma\rangle$.

where $N^{\prime}$ is given by $N / n, H_{0}^{(n)}$ is the trial Hamiltonian describing the sites $i$ and its neighbors $j$ of the cluster $N^{\prime}(i, j=1, \ldots, n)$.

\section{Numerical results and diagrams}

In this section, we present the phase diagram, magnetizations and the Gibbs free energy in order to find and study the Baxter ${ }^{(2)}$ phase. Once the Baxter ${ }^{(2)}$ phase is found we classify its phase transition with the others phases already known for the system. The spin-1/2 AT model presents different phases; here we make a brief description of those phases as a function of magnetizations $m_{\tau}$ (for more details of the description of the phases please see Ref. [6])

i) The Paramagnetic-phase labeled by "Para" where $m_{\tau}=0$.

ii) The $\langle\sigma\rangle$-phase denoted by “ $\langle\sigma\rangle$ ” where $m_{S}^{\prime}=0, m_{\sigma}^{\prime} \neq 0$ or $m_{S}^{\prime} \neq 0, m_{\sigma}^{\prime}=0$ and $m_{\sigma S}^{\prime}=0$.

iii) The $\langle\sigma S\rangle_{A F}$-phase indicated by " $\langle\sigma S\rangle_{A F}$ " where $m_{S}=0$, $m_{\sigma}=0$ and $m_{\sigma S} \neq 0$.

iv) The Baxter ${ }^{(1)}$-phase labeled by "Baxter ${ }^{(1)}$ " where $m_{S}=m_{\sigma} \neq 0$ and $m_{\sigma s} \neq 0$.

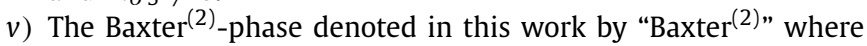
$m_{S}^{\prime \prime} \neq m_{\sigma}^{\prime \prime} \neq 0$ and $m_{\sigma S}^{\prime \prime} \neq 0$.

For the following discussions, let us define the parameters $x=$ $K / J$ and $T \equiv k_{B} T / J$.

We present in Fig. 2 results for the phase diagram as a function of $T$ and $x$ for the spin-1/2 AT model in a cubic lattice for the 1 -site cluster. The region for $x$ considered in this work shows the following phases of lower Gibbs free energy: Para, $\langle\sigma\rangle,\langle\sigma S\rangle_{A F}$, Baxter $^{(1)}$ and Baxter ${ }^{(2)}$. The second order phase transition lines between the phases Para-Baxter ${ }^{(1)}$, Para- $\langle\sigma\rangle$ and Para- $\langle\sigma S\rangle_{A F}$ are represented by solid lines and the first order phase transition line between the phases Baxter ${ }^{(1)}$ and $\langle\sigma\rangle$ is represented by dashed line. This phase transition was also obtained by other authors $[6$, $9,15,18]$. The dotted lines represent the value of the zero temperature and the boundary between the phases $\langle\sigma S\rangle_{A F}$ and $\langle\sigma\rangle$. Two other second order phase transitions were observed between the phases Baxter ${ }^{(1)}$-Baxter ${ }^{(2)}$ and Baxter ${ }^{(2)}-\langle\sigma\rangle$ and are represented by solid lines. Phase diagrams for 2-, 4- and 8-sites clusters were obtained and present similar characteristics as those obtained for the 1 -site cluster.

The magnetizations are illustrated in Fig. 3 as a function of $T$ for the spin-1/2 AT model in a cubic lattice for the 1 -site cluster, for a fixed $x=-0.8$. In the selected region limited by the points

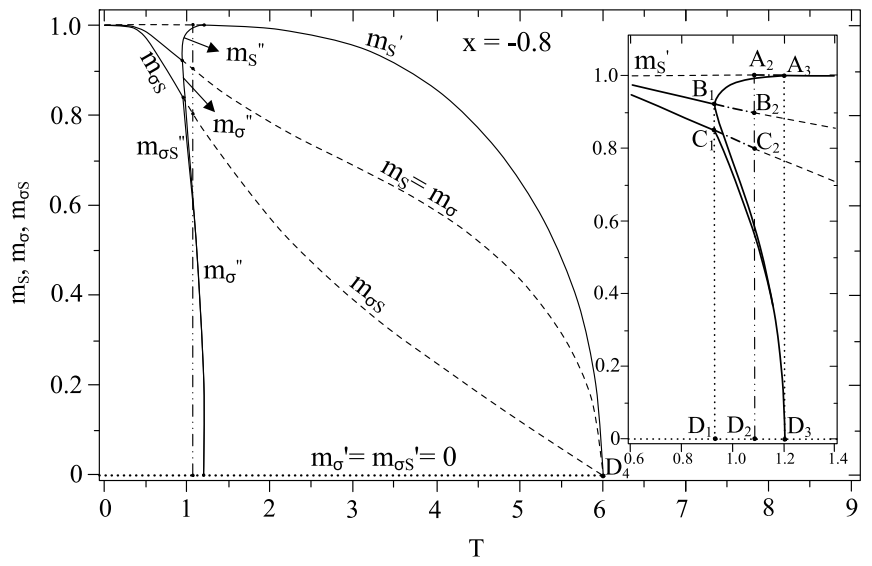

Fig. 3. The Magnetizations $\left(m_{S}, m_{\sigma}\right.$ and $\left.m_{\sigma S}\right)$ as a function of $T$ obtained for the spin-1/2 AT model in a cubic lattice with 1-site cluster from the MFT for fixed $x=-0.8$. The points $D_{1}, D_{2}, D_{3}$ and $D_{4}$ represents the values for $T$ where the phase transitions occurs.

\section{Table 1}

Numerical values of $T$ at the points $D_{1}, D_{2}, D_{3}$ and $D_{4}$ in the Fig. 3 for different clusters.

\begin{tabular}{lllll}
\hline Cluster & $D_{1}$ & $D_{2}$ & $D_{3}$ & $D_{4}$ \\
\hline 1-site & 0.936 & 1.084 & 1.204 & 6.000 \\
2-sites & 0.957 & 1.076 & 1.176 & 5.745 \\
4-sites & 0.980 & 1.069 & 1.148 & 5.462 \\
8-sites & 1.007 & 1.065 & 1.120 & 5.138
\end{tabular}

$D_{1}, D_{2}, D_{3}$ and $D_{4}$ that represent the values for $T$ where first and second order phase transitions occurs the model presents the following phases:

(a) In the region that goes from $T=0$ until $T=D_{1}$ the model presents the phases Baxter ${ }^{(1)}$ with magnetizations $m_{S}, m_{\sigma}$ and $m_{\sigma S}$ and the phase $\langle\sigma\rangle$ with magnetizations $m_{S}^{\prime}, m_{\sigma}^{\prime}$ and $m_{\sigma S}^{\prime}$.

(b) In the region between $D_{1}$ and $D_{3}$ the Baxter ${ }^{(1)}$ phase, $\langle\sigma\rangle$ phase and a new phase $\operatorname{Baxter}^{(2)}$ are present. This new phase presents the magnetizations $m_{S}^{\prime \prime}, m_{\sigma}^{\prime \prime}$ and $m_{\sigma S}^{\prime \prime}$, where $m_{S}^{\prime \prime}>$ $m_{\sigma}^{\prime \prime} \neq 0$. After the point $D_{1}$ we observed $m_{S}^{\prime \prime} \rightarrow m_{S}^{\prime}$ at $A_{3}$ and $m_{\sigma}^{\prime \prime}$, $m_{3}^{\prime \prime}$ going to zero at $D_{3}$. Therefore in the points $B_{1}$ and $C_{1}$ occurs a second order phase transition between the phases Baxter ${ }^{(1)}$ and Baxter ${ }^{(2)}$. The Baxter ${ }^{(2)}$ phase ends at the points $A_{3}$ and $D_{3}$ where a second order phase transition occurs between the phases Baxter $^{(2)}$ and $\langle\sigma\rangle$. We also observed a first order phase transition at the points $A_{2}, B_{2}, C_{2}$ at $T=D_{2}$ between the phases Baxter ${ }^{(1)}$ and $\langle\sigma\rangle$. This transition is in agreement with other works $[6,9,15$, $18]$.

(c) In the region between $D_{3}$ and $D_{4}$ we observe the phases $\operatorname{Baxter}^{(1)}$ and $\langle\sigma\rangle$ that present a second order phase transition at the point $D_{4}$.

Studying the effect of lattice size we found similar results for phase diagram, magnetizations and the Gibbs free energy obtained from the 1-site cluster. The numerical values of $T$ at the points $D_{1}$, $D_{2}, D_{3}$ and $D_{4}$ are presented in Table 1 for 1-, 2-, 4- and 8-site clusters. We observe that the new phase obtained in this work presents a decrease in the region between $D_{1}$ and $D_{3}$ with the increase of the size of the cluster, however, it is important to mentioned that the new phase was also found in the MC context for a considerable larger lattice comparing with the one used in this work [6]. There the authors also observed that the region where the new phase is present remains the same changing the size of the lattice. For more precise results about the effect of increasing the lattice size, please see Ref. [26]. Where the new phase can be studied under the effect of increasing the lattice size in the EFT context. 


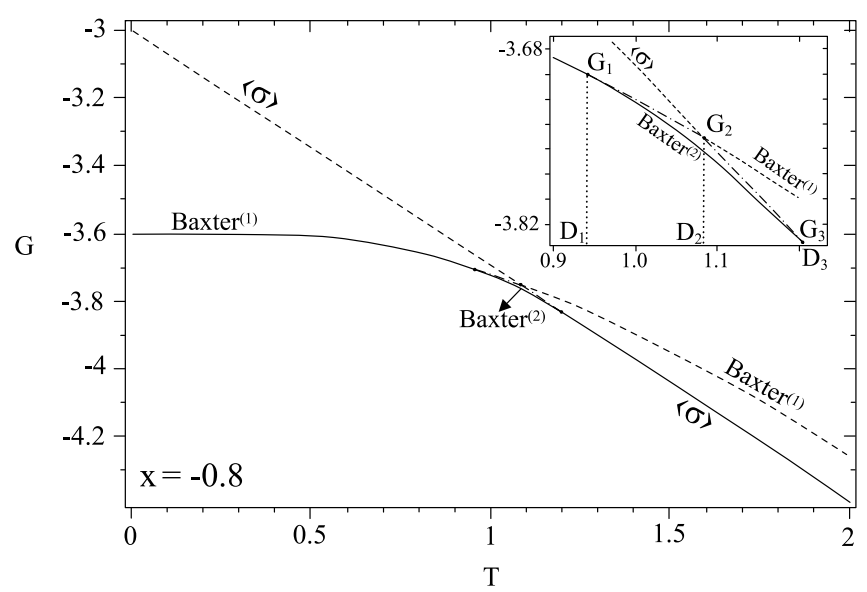

Fig. 4. The Gibbs free energy as a function of $T$ for fixed $x=-0.8$ obtained for the AT model from MFT considering 1-site cluster.

In Fig. 4 we present the Gibbs free energy as a function of $T$ for $x=-0.8$. From the Eqs. (10) and (11) the Baxter ${ }^{(1)}$, Baxter ${ }^{(2)}$ and $\langle\sigma\rangle$ phases were studied. In the dot-dashed line $G_{1} G_{2} G_{3}$ we have a discontinuity in the point $G_{2}$, a behavior characteristic of first order phase transitions. The solid line $G_{1} G_{3}$ has no discontinuity which is a characteristic of second order phase transitions. In the region between $T=0$ and $T=D_{2}$ the $\langle\sigma\rangle$ phase presents energy greater than the Baxter ${ }^{(1)}$ phase. At the point $D_{1}$ a new phase emerges, labeled in this work by $\operatorname{Baxter}^{(2)}$. This new phase presents energy smaller than the phases $\operatorname{Baxter}^{(1)}$ and $\langle\sigma\rangle$, that is, there is a second order phase transitions between the phases Baxter $^{(1)}$ and Baxter ${ }^{(2)}$ until the point $G_{3}$ where a second order phase transition occurs between the phases Baxter ${ }^{(2)}$ and $\langle\sigma\rangle$. We also found the first order transition between the phases $\operatorname{Baxter}^{(1)}$ and $\langle\sigma\rangle$ at the point $G_{2}$. In the region between the points $G_{1} G_{2} G_{3}$ we have two situations: First, $G_{1} G_{2}$ with the energy of $\langle\sigma\rangle$ greater than the Baxter ${ }^{(1)}$ and second $G_{2} G_{3}$ with the energy of $\operatorname{Baxter}^{(1)}$ greater than $\langle\sigma\rangle$. It is also possible to observe that both $\operatorname{Baxter}^{(1)}$ and $\langle\sigma\rangle$ have energy greater than $\operatorname{Baxter}^{(2)}$ in the region $G_{1} G_{2} G_{3}$.

In the Ref. [6] Ditzian et al. observed from MC simulations that for $-0.75>x>-0.78$ an unexpected behavior occurs. The system seems to have both $\langle\sigma\rangle$ and $\langle S\rangle$ different from zero but $\langle\sigma\rangle \neq\langle S\rangle$. The system presents two fixed values for the order parameters $|\langle\sigma\rangle|$ and $|\langle S\rangle|$ with $|\langle S\rangle|>|\langle\sigma\rangle|$. Moreover they also observed that if there is really a range in which $m_{S}>m_{\sigma}>0$, this is a new phase not seen in MFT at that time. The transitions between the phases Baxter ${ }^{(1)}$ and $\langle\sigma\rangle$ were also classified like first order transitions by Musial [18] (he observed a small presence of latent heat in this region). However, in the present work we have shown the existence of the new Baxter ${ }^{(2)}$-phase, where $m_{S} \neq m_{\sigma} \neq 0$. In addition, the new phase transitions were classified in the region $-1>x>-0.5$.

\section{Final comments}

We have presented the MFT results obtained from the Bogoliubov inequality for the spin-1/2 AT model on the cubic lattice. The free energy, magnetization and critical frontiers are obtained for different cluster sizes. Studying the phase diagram in the region $-1<x<-0.5$ we observed a new phase never studied before. The Baxter ${ }^{(2)}$-phase, as denoted in this article, presents the following properties: first $\langle S\rangle \neq\langle\sigma\rangle \neq 0$ and $\langle\sigma S\rangle \neq 0$, studied in details in the region $x=-0.8$ and second it presents a second order phase transition with the phases $\operatorname{Baxter}^{(1)}$ and $\langle\sigma\rangle$. In the interval that was found this new phase we also observed the phases Baxter $^{(1)} \quad(\langle S\rangle=\langle\sigma\rangle \neq 0$ and $\langle\sigma S\rangle \neq 0)$ and $\langle\sigma\rangle \quad(\langle S\rangle=\langle\sigma\rangle=0$ and $\langle\sigma S\rangle \neq 0$ ) with higher free energy. The first order phase transition between these two phases was also obtained and is in agreement with other authors $[6,9,15,18]$.

\section{Acknowledgements}

Work was partially financed by Conselho Nacional de Desenvolvimento Científico e Tecnológico-CNPq Grant No. 142029/ 2017-3 (D.S.R).

\section{References}

[1] J. Ashkin, E. Teller, Phys. Rev. 64 (1943) 178.

[2] L.F. Feiner, A.M. Oles, Phys. Rev. B 59 (1999) 3295.

[3] M. Sluiter, Y. Kawazoe, Sci. Rep. RITU A 40 (1995) 301.

[4] C. Zhe, W. Ping, Z. Ying-Hong, Commun. Theor. Phys. 49 (2008) 525.

[5] P. Bak, P. Kleban, W.N. Unertl, J. Ochab, G. Akinci, N.C. Bartelt, T.L. Einstein, Phys. Rev. Lett. 54 (1985) 14.

[6] R.V. Ditzian, R.J. Banavar, G.S. Grest, L.P. Kadanoff, Phys. Rev. B 22 (1980) 2542.

[7] Ü. Akinci, Physica A 469 (2017) 740.

[8] P.L. Christiano, S. Goulart Rosa Jr., Phys. Rev. A 34 (1986) 730.

[9] J.P. Santos, F.C. Sá Barreto, Physica A 421 (2015) 316.

[10] J.P. Santos, F.C. Sá Barreto, Braz. J. Phys. 46 (2016) 70.

[11] J.A. Plascak, F.C. Sá Barreto, Physica A 19 (1986) 2195.

[12] P.M.C. Oliveira, F.C. Sá Barreto, J. Stat. Phys. 53 (1989) 57.

[13] P. Pawlicki, G. Musial, G. Kamieniarz, J. Rogiers, Physica A 242 (1997) 281

[14] N. Benayad, A. Benyoussef, N. Boccara, A.El. Kenz, J. Phys. C 21 (1988) 5747.

[15] P. Arnold, Y. Zhang, Nucl. Phys. B 501 (1997) 803.

[16] D. Jeziorek-Kniola, G. Musial, L. Debski, J. Rogiers, S. Dylak, Acta Phys. Pol. A 1105 (2012) 121

[17] G. Musial, L. Debski, G. Kamieniarz, Phys. Rev. B 66 (2002) 012407.

[18] G. Musial, Phys. Status Solidi B 236 (2003) 486.

[19] G. Musial, Phys. Rev. B 69 (2004) 024407.

[20] G. Musial, J. Rogiers, Phys. Status Solidi B 243 (2006) 335.

[21] P. Pawlicki, G. Musial, G. Kamieniarz, J. Rogiers, Physica A 242 (1997) 281.

[22] N.N. Bogoliubov, J. Phys. USSR 11 (1947) 23.

[23] R.P. Feynman, Phys. Rev. 97 (1955) 660.

[24] H. Falk, Am. J. Phys. 38 (1970) 858.

[25] J.P. Santos, Braz. J. Phys. 1 (2017) 1.

[26] Ü. Akinci, J. Magn. Magn. Mater. 386 (2015) 60. 